\title{
Pregnancy in a didelphic uterus: a case report of an unusual phenomenon and review of literature
}

\author{
Emmanuel Ekanem*, Lalrinawmi Lalrinawmi, Anita Sinha, Tamer Abdelrazik
} Department of Obstetrics and Gynaecology, Great Western Hospital NHS Foundation Trust, Swindon, SN3 6BB,
United Kingdom

Received: 28 December 2020

Accepted: 04 February 2021

*Correspondence:

Dr. Emmanuel Ekanem,

E-mail: Emmanuel.Ekanem@nhs.net

Copyright: (C) the author(s), publisher and licensee Medip Academy. This is an open-access article distributed under the terms of the Creative Commons Attribution Non-Commercial License, which permits unrestricted non-commercial use, distribution, and reproduction in any medium, provided the original work is properly cited.

\begin{abstract}
Mullerian anomalies are defects in the embryological development of the urogenital systems as these organs begin to form at about the 5 and 6th week of intrauterine life. Uterine didelphys is one of the Mullerian or paramesonephric duct anomalies occurring as a result of duplication of the uterine canal with two cervical canals and a single vagina. The incidence uterine didelphys has been quoted as 1 in 2000 women. This case report elucidates the presentation of a multiparous lady with uterine didelphys who had a previous successful pregnancy in of the uterine canals but presented in the second pregnancy with a missed miscarriage at 12 weeks gestational age. She also had a single right kidney. These were all diagnosed on ultrasound scan. She had an initial unsuccessful surgical management of miscarriage with uterine perforation of one of the uterine cavities diagnosed on hysteroscopy and diagnostic laparoscopy. She subsequently had examination under anaesthesia and an attempt at evacuation of retained products and a second look laparoscopy. This was particularly challenging as the cervix was flushed with the vaginal vault making delineation and dilatation of the cervical OS difficult hence sustaining a perforation at the left uterine cavity. The second attempt of surgical management was successful, and she was subsequently discharged. This is one of the few case reports in literature and will further add credence to the body of knowledge of a different aspect to the presentation and management of cases of Mullerian anomalies.
\end{abstract}

Keywords: Didelphys, Uterus, Mullerian, Anomalies, Pregnancy

\section{INTRODUCTION}

Disorders of the Mullerian system are unusual occurrences and usually present with different symptoms to the paediatric and adolescent gynaecologist. They normally occur as result of abnormality in the development of the female genital tract during intrauterine life. ${ }^{1,2}$ They involve the entire urogenital system consisting of the urinary system, the vagina, cervix, uterus and fallopian tubes and may present as imperforate hymen with haematocolpus, severe menstrual pain, miscarriages and others may have bearing with fertility. ${ }^{3}$ Due to the close proximity of the urinary system and the genital embryological development, maldevelopment involving the kidneys and the collecting duct system may also be involved. ${ }^{4-6}$ Management usually involves a multi-professional team of paediatric and adolescent gynaecologist, obstetricians, psychologist, geneticist and radiologist. ${ }^{3}$

\section{CASE REPORT}

This is the case presentation of a 35-year-old G2P1 lady of 12 weeks gestational age who was referred by her general practitioner with symptoms of vaginal bleeding, which was dark brown in nature, not copious and with associated minimal crampy abdominal pain. There was no dizziness or fainting spells, urinary or bowel symptoms. 
The index pregnancy was quite uneventful until the bleeding started. For the past obstetric history, she had an undiagnosed breech presentation in her last pregnancy and had an uneventful elective caesarean section and uterine didelphys was confirmed at surgery with the foetus in the right uterine cavity. She had an uneventful past gynaecological history with no history of hypomenorrhea, menorrhagia or dyspareunia.

In her past medical history, she was discovered to have a single right kidney incidentally noticed on ultrasound and has been constantly followed up by the nephrologist with no complications ensuing.

On examination when she presented, she was not in any form of distress and her observations were all normal.

The abdomen soft with minimal suprapubic tenderness and pelvic examination was unremarkable.

Investigations done included a complete blood count which showed haemoglobin of $142 \mathrm{~g} / \mathrm{l}$, total white cell count of $4.8 \times 10^{9} / 1$ and platelets of $151 \times 10^{9} / 1$ and her electrolytes were normal.

A transvaginal ultrasound scan revealed a didelphic uterus with 1 cervix and 1 vagina, and a well-formed intrauterine pregnancy seen in the left uterine cavity, CRL-14.5 mm 7 weeks 5 days with no foetal heart pulsations identified using colour Doppler. This was confirmed by a second sonographer. The right ovary was of a normal appearance and the left ovary was not identified. The impression made from the ultrasound scan was a missed miscarriage.

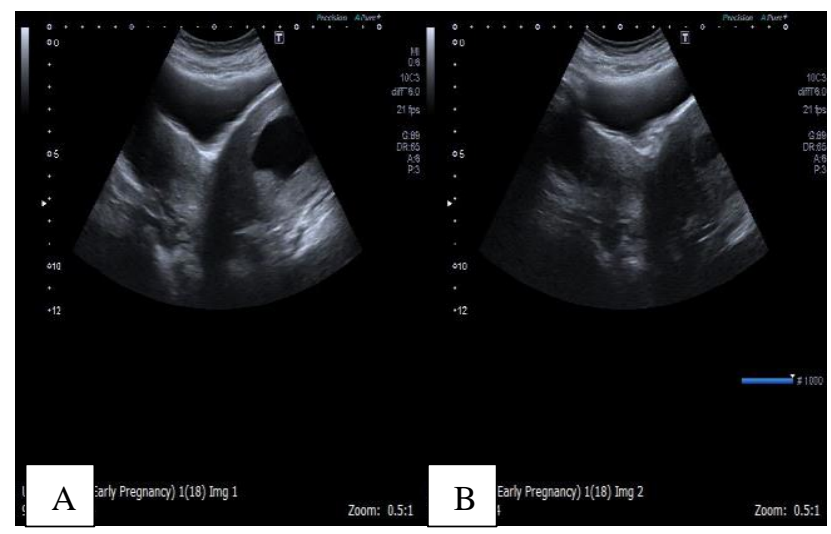

Figure 1: The left image of the left and right uterine cavities with the pregnancy in the left cavity and the bladder superior to them.

She was counselled on the options of management which were expectant, medical and surgical and she preferred the medical option. She had the medical management with misoprostol but however the medical management failed. She had a repeat scan which revealed: a single gestation sac seen within the upper left uterus. Foetal pole again seen. $\mathrm{CRL}=13 \mathrm{~mm}$ with no foetal heart motion seen. Normal appearances of the right uterus. The impression made was appearances consistent with an incomplete miscarriage.



Figure 2: Both images of the incomplete miscarriage.

Based on the failed medical management she was further counselled on options of treatment and she chose surgical management of miscarriage plus a hysteroscopy. She was subsequently prepared, and she had surgical management of miscarriage with hysteroscopy. The intraoperative findings were normal vulva and vagina, cervix flushed with the vault, two cervical OS and a bulky 12 weeks size uterus. The cervical canals could not be easily delineated making dilatation of the cervical OS very challenging. Attempt at the dilatation of the most obvious cervical OS went straight into the right dominant uterus, which was easier to dilate and unfortunately, she had fundal uterine perforation and the perforation was confirmed on hysteroscopy. A diagnostic laparoscopy was also done and the perforation in the right uterine fundus was also confirmed and coagulated with bipolar diathermy.

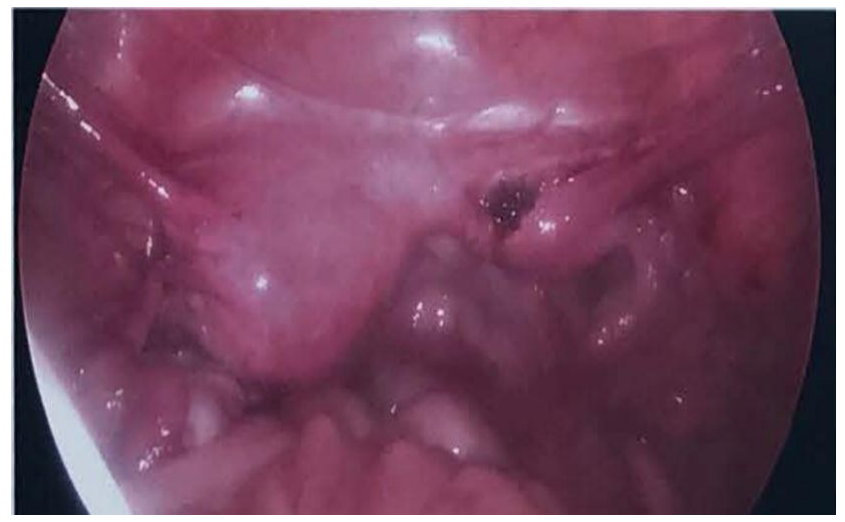

Figure 3: Diagnostic laparoscopy and right uterine perforation confirmed with normal tubes and ovaries attached to each uterus.

The left cervical OS was not easily discernible due to its acute curvature into the uterine cavity and several attempts at dilatation under ultrasound and laparoscopic guidance was unsuccessful and the procedure abandoned. She was debriefed and counselled on options including 
conservative, medical and surgical and after thoughtful consideration she chose the surgical option. She was prepared for examination under anaesthesia, attempt at evacuation of retained products and if not successful then laparoscopy plus hysterotomy and evacuation of the uterus. She had a successful examination under anaesthesia and evacuation of retained products under ultrasound guidance and a check laparoscopy afterwards. The intraoperative findings were: normal vulva and vagina, cervix flushed with the vault, two cervical OS both dilated, bulky 12 weeks size uterus. This was also done ultrasound scan guidance and the left uterine cavity was confirmed to be empty. Check laparoscopy was also done and there was no bleeding from the site of perforation and also showed a reduction in the size of the left uterine cavity.

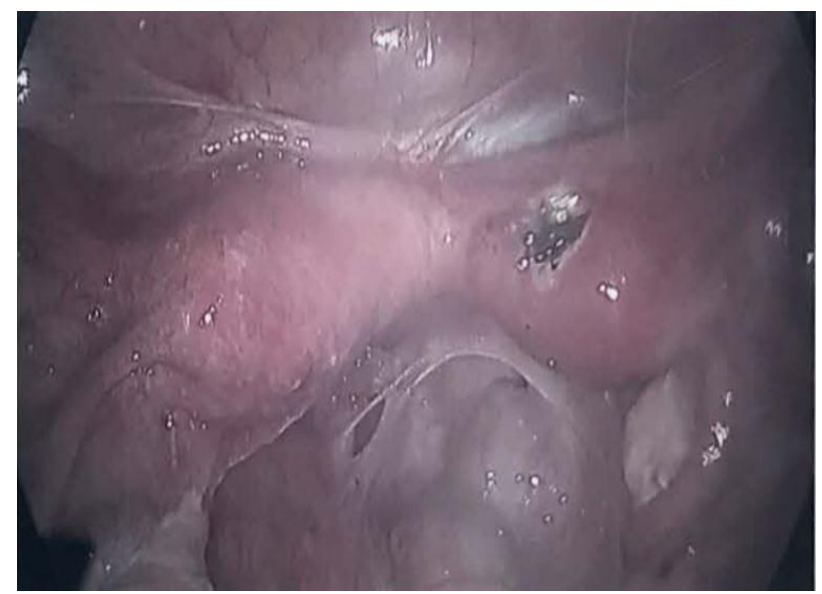

Figure 4: Check laparoscopy with no further bleeding from perforated right uterus and reduction in the size of left uterine cavity.

Postoperatively she had an uneventful recovery and received intravenous antibiotics for 24 hours, and then orals for 7 days thereafter. She was adequately debriefed of the events and all concerns were addressed. She was discharged home on the second postoperative day and given a 3 month follow appointment in the general outpatient department.

\section{Challenges}

This case presented was not a straightforward case as patents who present with uterine didelphys usually have a longitudinal vaginal septum with discernible cervical canals. ${ }^{1}$ The challenges that were faced in this case were:

The cervical canals not being obvious and not easily delineated making dilatation of the cervical OS very challenging. Attempt at the dilatation of the cervical OS went straight into the right dominant uterine cavity which was easier to dilate and unfortunately a fundal uterine perforation occurred.

The left cervical OS was not so conspicuous and the cervix was flushed with the vagina and due to the acute curvature of the cervical canal into the uterine canal, several attempts at dilatation under ultrasound and laparoscopic guidance was futile hence a failed attempt at surgical evacuation. The previous attempt at dilatation and surgical evacuation with ongoing bleeding may have wided the canal more making the second attempt at evacuation successful.

\section{DISCUSSION}

Mullerian anomalies are among a spectrum of disorders affecting the genital and urinary system as they both share a common embryological origin. The classification widely accepted is the that of the American fertility society and the clinical embryological classification by Acien and colleagues. ${ }^{7,8}$

All patients with uterine didelphys usually present with a longitudinal vaginal septum and rarely do patients present without a vaginal septum. ${ }^{9}$ This was a deviation from the norm as the index patient did not present with a vaginal septum.

\section{Embryology}

The urinary and the genital systems are closely related embryologically and both derive their origin from the intermediate mesoderm and the cloaca. ${ }^{10}$ The intermediate mesoderm during its development between the paraxial mesoderm and the lateral plate mesoderm forms a bulge on the posterior abdominal wall known as the urogenital ridge. ${ }^{10}$ The urogenital ridge divides into a medial and lateral part. The medial part forms the genital system and the lateral part forms the urinary system. ${ }^{10}$

During embryogenesis, the genital system begins its development from about the $5^{\text {th }}$ and $6^{\text {th }}$ week of intrauterine life and at this stage it is said to be in an indifferent state. ${ }^{4,5}$ The Mullerian duct or the paramesonephric duct is responsible for the development of the female genital tract. ${ }^{4}$

Oestrogen is responsible for the Mullerian duct development in the absence of the anti-Mullerian hormone $(\mathrm{AMH}){ }^{4}$

The Mullerian duct has 3 parts, the cranial potion which opens into the peritoneal cavity, the part that crosses the Wolffian duct and the lower part which fuses with the duct of the contralateral side. ${ }^{5}$

The paramesonephric duct or the Mullerian duct lies lateral to mesonephric duct which is also known as the Wolffian duct. The paramesonephric duct crosses to the medial side of the mesonephric duct and the ducts of the two sides meet at the midline giving rise to the uterovaginal canal or the uterine canal. ${ }^{5,10}$ The paramesonephric duct also gives origin to the fallopian tubes, cervix and the vagina. ${ }^{10}$ The uterus develops from the upper fused portion of the Mullerian ducts while the 
myometrium forms from the surrounding mesoderm. ${ }^{10}$ The cervix is also derived from the Mullerian duct and is usually larger than the body of the uterus at birth. ${ }^{10}$ Due to the close relationship between the urinary and genital systems, abnormalities of both systems usually coexist.

\section{Prevalence}

The incidence of these anomalies is not easily determined as patients do not normally present except when symptoms heralds. Using imaging modalities to determine the incidence of Mullerian anomalies has also shown a divide and a bias in certain women presenting with symptoms and these imaging modalities such as hysteroscopy or hysterosalpingogram tend to overemphasize the incidence. ${ }^{11}$ Also, there is a variation in incidence in fertile and infertile women and in women who present with first and second trimester miscarriages and recurrent miscarriages. ${ }^{11-13}$

The incidence varies from 1-5\%.1,11,13-15 The prevalence of uterine didelphys has been cited as 1 in 2000 women in literature. ${ }^{1}$

\section{Types and clinical presentation}

The widely accepted classification system of Mullerian anomalies is the classification by the American fertility society and Acien et al clinical embryological classification. $^{7,8}$

The American fertility society classifies is it into seven classes as shown in table 1.

Table 1: The American fertility society classification of Mullerian anomalies.

\begin{tabular}{|ll|}
\hline Classification & \\
\hline Class I & Hypoplasia/agenesis \\
\hline Class II & Unicornuate uterus \\
\hline Class III & Didelphys \\
\hline Class IV & Bicornuate \\
\hline Class V & Septate uterus \\
\hline Class VI & Arcuate \\
\hline Class VII & Diethylstilboestrol drug related. ${ }^{8}$ \\
\hline
\end{tabular}

This classification has its emphasis on the defects in the vertical unification of the Mullerian duct as opposed to the classification by Acien and colleagues which emphasised on the pattern of development and clinical presentation. $^{3}$

The various anomalies of the uterus include septate uterus which is by far the commonest in $35 \%$ of cases. ${ }^{13}$ This occurs as a result of lack of resorption of the internal uterine septum between the two uterine cavities in the presence of complete lateral fusion of the paramesonephric duct giving the normal appearance of the surface of the uterus. ${ }^{11}$ Here, the uterus and vagina may be maybe separated in two by a septum. ${ }^{5}$ Septate uterus can be complete or complete depending on the varying degree of resorption of the septum.

Bicornuate uterus is one of the commonest anomalies after septate uterus and occurs in $5-30 \%$ of cases. ${ }^{13}$ This occurs when the paramesonephric ducts fail to unite completely resulting in varied intensity of separation of the two uterine cavities. ${ }^{4,11,13,16}$ The mildest form of separation may present as arcuate uterus and in the severest form it may present with two uterine horns that are separated up to the internal OS with no link or communication between the two cavities. ${ }^{11}$ Partial bicornuate uterus is variant between the two as described above with great indentation involving the two horns of the uterus and a lateral fusion with central cavity before the cervical internal OS. ${ }^{11}$ If the development of the Mullerian duct is inhibited and does not unite with the second portion then this becomes a bicornuate uterus with a rudimentary horn and this may not communicate with the uterine cavity. ${ }^{4}$

Arcuate uterus is another common variety of the Mullerian duct system anomalies and here the uterus may be indented in the middle. This occurs in about $20 \%$ of cases. $^{5,13}$ This is been associated with recurrent miscarriages.

When one Mullerian duct fails to develop then we have a unicornuate uterus and this gives rise to a uterus with one fallopian tube. ${ }^{4}$ There are several varieties of the unicornuate uterus ranging from the an isolated unicornuate uterus with no contralateral structure or one with either an anlage, or one where a rudimentary horn is present contralateral to the unicornuate uterus. ${ }^{11}$ The rudimentary horn may or may not be communicating with the uterine cavity. ${ }^{11}$ Unicornuate uterus has a predilection for preterm labour and recurrent miscarriages. ${ }^{4}$

Uterine didelphys or a double uterus occurs from inability of the inferior or lower portion of the Mullerian duct to unite. This results in complete duplication of the uterus with two cervical canals and a single vagina. ${ }^{1,4,11}$ This is sometimes said to be restricted to the uterus and the cervix but may also involve duplication of the vulva, vagina, urethra, urinary bladder and the anus. ${ }^{11}$ Some patients with uterine didelphys may not present with any problems but others may present with obstructed hemivagina with ipsilateral renal anomaly. ${ }^{11}$ All Patients with uterine didelphys usually present with a longitudinal vaginal septum and rarely do patients present without a vaginal septum. ${ }^{9}$

Women with uterine didelphys will go on to have a normal birth with a delivery rate at term estimated to be about $40 \% .^{13}$

\section{Clinical presentation}

Patients with Mullerian duct anomalies may not present with symptoms and this may only be picked up on routine 
examination for another condition. Typical presentation usually include menstrual abnormalities such as dysmenorrhea or pelvic pain as a result of haematolpus from an imperforate hymen, haematometria, hypomenorrhea, endometriosis, miscarriages and infertility. ${ }^{11}$

\section{Diagnosis}

Diagnosis of Mullerian anomalies is usually suspected from clinical case presentation and confirmation is by imaging modalities. Several imaging modalities have been employed ranging from 2D to 3D ultrasound scan and MRI. ${ }^{17}$ Ultrasound provides an invaluable resource in delineating the urogenital system and identifying normal and abnormal anatomy. This can be transvaginal, transabdominal or transperineal. ${ }^{18}$

Magnetic resonance imaging has become the gold standard in the diagnosis of uterine anomalies as it is very sensitive and specific and very relevant in defining the different forms of uterine anomalies involving uterine horns and renal anomalies. ${ }^{19}$ Other modalities that have also proven to be helpful include, hysterosalpingogram, hysteroscopy and laparoscopy. ${ }^{13,20}$

The case presented is that of a 35-year G2P1 of 12 weeks gestational age who presented with symptoms of vaginal bleeding and pelvic pain in keeping with a miscarriage. She was diagnosed with uterine didelphys in her previous pregnancy where she had a caesarean section due to an undiagnosed breech presentation in the left uterine cavity. In the index pregnancy, she had surgical management of miscarriage after a previous unsuccessful attempt. This was fraught with challenges as numerated above as they were very peculiar and unusual and not routinely encountered in these patients.

Patients are usually asymptomatic and present either with symptoms or the anomaly discovered on routine gynaecological examination and imaging. Symptoms include vaginal bleeding, pelvic pain from haematocolpus or from an imperforate hymen, dysmenorrhea, dyspareunia or infertility. This is said to be more in patients who present with a vaginal septum. ${ }^{1}$ Heinonem in his report of uterine didelphys: a case report of 26 cases revealed that the most common presenting symptoms in the 26 patients were dysmenorrhea, dyspareunia and leukorrhea. ${ }^{1}$ Some patients may also present with the syndrome OHVIRA known as obstructed hemivagina and ipsilateral renal anomaly. ${ }^{21}$ This was first reported in the early twentieth century and referred to Herlyn-Werner-Wunderlich syndrome. ${ }^{21}$ This is an unusual and infrequent anomaly affecting both the genital and the urinary system and patients normally present with the characteristic symptoms of obstruction of hemivagina, ipsilateral renal agenesis and uterine didelphys. This has been reported in few case studies..$^{21,22}$
Due to the proximity in the development of the urogenital system, there may be concomitant abnormalities of the urinary system. The index patient had a single right kidney and was under the follow up of her nephrologist and did not have any complication or sequalae. Renal agenesis has been noted to occur and this is seen in about two percent of cases of Mullerian duct anomalies. ${ }^{1}$ Maren and associates in their case report also reported the occurrence of uterine didelphys with associated unilateral renal agenesis in both mother and foetus as picked up at routine 20 weeks anomaly scan. ${ }^{6}$ Cases of absent kidneys have also been reported. ${ }^{1}$

Different pregnancy outcomes have been reported in patients with Mullerian anomaly. The index patient presented with 12 weeks missed miscarriage in the right uterine cavity. Sotirios and colleagues evaluated the pattern of pregnancy loss in women with congenital uterine anomalies and recurrent miscarriages. They revealed that patients with bicornuate or septate uterus had a significant increase in first trimester losses as well as second trimester miscarriage compared to the control group. ${ }^{12}$ Raga and colleagues also noted that women with uterine didelphys are more prone to miscarriages and preterm deliveries compared to the general population. ${ }^{23}$ The rate of spontaneous pregnancy loss in women with Mullerian anomalies as high as $60 \%$ has been cited by various studies. ${ }^{24-27}$ Also Christos and associates in their paper on clinical implications of uterine anomalies: a meta-analysis of comparative studies found that women with congenital uterine anomalies were more likely to be at risk of miscarriages (RR $1.68,95 \%$ CI 1.31 to 2.15 ). ${ }^{28}$ First trimester miscarriages is said to be more in women with septate and biconuate uterus compared to arcuate and unicornuate uterus. $^{28}$ With regards to second trimester miscarriage, women with didelphys uterus and unicornuate uterus are less likely to be at risk of this compared to arcuate, septate and bicornuate uterus. ${ }^{28}$

There are numerous cases reports replete with successful pregnancies in women with uterine didelphys and some stating a decrease in pregnancy rate in these women too. Women with uterine anomalies have different life birth rates and this has been cited in studies. ${ }^{13}$ Grimbizis and colleagues in their work on clinical implications of uterine malformations and hysteroscopic treatment results discovered that rate of term delivery in women with Mullerian anomaly was approximately fifty percent and these women were also noted to have more complications in pregnancy, labour and delivery. ${ }^{13}$ Also women with uterine didelphys and unicornuate uterus had a delivery rate at term of approximately forty percent. Women with arcuate uterus were known to have the highest delivery rate of sixty five precent. ${ }^{13}$ Heinonem in his case report revealed a foetal survival rate of $67 \%$ and a perinatal mortality rate of $3.6 \% .^{1}$

Also successful singleton twin and triplet gestations have been reported in patients with uterine didelphys. ${ }^{17,29-32}$ 
The index patient has had a previous normal term delivery in the left uterine cavity and was delivered by caesarean section due to undiagnosed breech presentation at term. Heinonem reported a breech presentation rate of $43 \%$ and a caesarean section rate of $82 \%$ in his case report of 26 cases of women with uterine didelphys. ${ }^{1}$

On the contrary others have noted that pregnancy and livebirths are less likely in women with didelphys uterus. Raga and associates noted that women with uterine didelphys have the least likelihood of having term deliveries compared to women with other Mullerian anomalies. $^{23}$ This has also been corroborated by other reports and studies. ${ }^{13,33}$ This has also been emphasised by Acien in his publication of reproductive performance in women uterine malformation. ${ }^{34}$

Ectopic pregnancy has also been reported in patients with didelphys uterus and Chitra reported a case of cornual and cervical ectopic pregnancy diagnosed on ultrasound scan and confirmed with magnetic resonance imaging. ${ }^{35}$

Complications of pregnancy such as foetal growth restriction, preterm births, malpresentation, abruption, retained placenta, operative births been noted in patients with Mullerian anomalies. ${ }^{23,24,36}$ These complications are thought to be due to either a reduction in the mass of the uterine myometrium, aberrant or abnormal blood flow within the uterus or cervical insufficiency. ${ }^{11}$ Christos et al in their metanalysis of clinical implications of uterine anomalies stated that women with congenital uterine anomalies were more likely to have preterm births and malpresentation and this risk was the same in unicornuate, arcuate, septate, bicornuate and uterine didelphys. ${ }^{28}$ Intrauterine growth restriction is said to be more in women with septate and uterine didelphys compared to other anomalies. ${ }^{28}$ Women with septate and arcuate uterus are at more risk of abruption placenta compared to uterine didelphys and others. ${ }^{28}$ With regards to perinatal mortality, women with septate and bicornuate uterus are more likely to have perinatal mortality. This was also the case with uterine didelphys, arcuate and unicornuate but this was not statistically significant. ${ }^{28}$

Various treatment options to improve pregnancy outcome have been tried with comparative success. Although there are conflicting reports of five percent pregnancy and live birth rate, Grigoris and associates in their study revealed that at term the rate of successful delivery was approximately seventy five percent and a live birth rate of approximately eighty five percent in women who had undergone hysteroscopic resection of the Mullerian anomaly. ${ }^{13}$ This seems very promising for patients in improving pregnancy and live birth rates.

\section{CONCLUSION}

In conclusion, disorders of the Mullerian duct system are rare occurrences and could also affect the urinary system due their close embryological origin. Uterine didelphys or double uterus is one of the anomalies that presents to the gynaecologist. Patients are usually asymptomatic with the anomalies noticed on routine examination and imaging for other reasons. For the proportion of women who present with symptoms, these include dyspareunia, dysmenorrhea suprapubic pain, haematocolpus or haematmetria and miscarriages. Diagnosis is usually with the help of a pelvic ultrasound scan and other diagnostic aids include magnetic resonance imaging as gold standard, hysterosalpingogram, hysteroscopy and laparoscopy. Pregnancy outcome is said to be promising with successful treatment. At present, there are only a few case reports on uterine didelphys and its presentation, and more studies are needed to evaluate and improve pregnancy and live birth rate.

Funding: No funding sources

Conflict of interest: None declared

Ethical approval: Not required

\section{REFERENCES}

1. Heinonen PK. Uterus didelphys: a report of 26 cases. Eur J Obstetr Gynecol Reprod Biol. 1984;17:345-50.

2. Rezai S, Bisram P, Lora Alcantara I, Upadhyay R, Lara C, Elmadjian M. Didelphys Uterus: A Case Report and Review of the Literature. Case Rep Obstet Gynecol. 2015;2015:1-5.

3. Edmonds DK, Rose GL. Outflow tract disorders of the female genital tract. Obstet Gynaecol. 2013;15(1):11-7.

4. Keith L More, T.V.N. Persaud MGT. The Developing Human Clinically Oriented Embryology. Developing Human Brain. 2016;524.

5. Sadler TW. Langman's Medical Embryology, Lippincott Williams and Wilkins, $14^{\text {th }}$ Edition. 2019;209.

6. Boehnke M, Zaretsky MV, Soranno DE, Dannull KA, Tucker BD, Marwan AI. Uterine didelphys with concomitant renal anomalies in both mother and fetus. J Pediatr Surg Case Reports. 2016;14:19-21.

7. Acien P, Acien M S-FM. Complex malformations of the female genital tract. New types and revision of classification. Hum Reprod. 2004;19:2377-84.

8. The American Fertility Society classifications of adnexal adhesions, distal tubal occlusion, tubal occlusion secondary to tubal ligation, tubal pregnancies, Müllerian anomalies and intrauterine adhesions. Fertil Steril. 2016;49(6):944-55.

9. Heinonen PK. Longitudinal vaginal septum. Eur J Obs Gynec reprod Biol. 1982;13:253-8.

10. Subhadra Devi V. Inderbir Singh's Human Embryology, Jaypee Brothers Medical Publishers; $11^{\text {th }}$ edition. $2018 ; 362$.

11. Reichman DE, Laufer MR. Congenital uterine anomalies affecting reproduction. Best Pract Res Clin Obstet Gynaecol. 2010;24(2):193-208.

12. Saravelos SH, Cocksedge KA, Li TC. The pattern of pregnancy loss in women with congenital uterine anomalies and recurrent miscarriage. Reprod Biomed 
Online. 2010;20(3):416-22.

13. Grimbizis GF, Camus M, Tarlatzis BC, Bontis JN, Devroey P. Clinical implications of uterine malformations and hysteroscopic treatment results. Human Reproduction Update. 2001;7:161-74.

14. Simon C, Martinez L, Pardo F, Tortajada M, Pellicer A. Mullerian defects in women with normal reproductive outcome. Fertil Steril. 1991;56(6):11923.

15. Jurkovic D, Gruboeck K, Tailor A, Nicolaides KH. Ultrasound screening for congenital uterine anomalies. BJOG An Int J Obstet Gynaecol. 1997;104(11):1320-1.

16. Letterie GS. Management of congenital uterine abnormalities. Reprod Biomed Online. 2011;23(1):40-52.

17. Rezai S, Bisram P, Alcantara IL, Upadhyay R, Lara C, Malvina E. Didelphys Uterus: A Case Report and Review of the Literature. Case Rep Obstet Gynecol. 2015;1-5.

18. Nicolini U, Bellotti M BB. Can ultrasound be used to screen uterine malformations? Fertil Steril. 1987;47(1):89-93.

19. Pellerito JS, McCarthy SM DM. Diagnosis of uterine anomalies: relative accuracy of MR imaging, endovaginal sonography, and hysterosalpingography. Radiology. 1992;183(3):795-800.

20. Reuter KL. Septate versus bicornuate uteri: errors in imaging diagnosis. Radiology. 1989;172(3):749-52.

21. Smith NA LM. Obstructed hemivagina and ipsilateral renal anomaly (OHVIRA) syndrome: management and follow-up. Fertil Steril. 2007;87:918-22.

22. Mandava A. OHVIRA Syndrome (obstructed hemivagina and ipsilateral renal anomaly) with Uterus Didelphys, an Unusual Presentation. J Pediatr Adolesc Gynecol. 2012;25(2):e23-5.

23. Raga F, Bauset C, Remohi J, Bonilla-Musoles F, Sim'on C. Reproductive impact of congenital Mullerian anomalies. Hum Reprod. 1997;12(10):2277-81.

24. Green LK, Harris RE. Uterine anomalies: frequency of diagnosis and associated obstetric complications.
Obs GynecoL. 1976;47(4):427-9.

25. Harger JH. Etiology of recurrent pregnancy loss and outcome of subsequent pregnancies. Obs Gyneco. 1983;62(5):574-81.

26. Heinonen PK. Reproductive performance ofwomen with uterine anomalies. Acta Obs Gynecol Scand. 1982;61(2):157-62.

27. Golan A, Langer R BI, Bukovsky I, Caspi E. Congenital anomalies of the mullerian system. Fertil Steril. 1989;51(5):747-55.

28. Venetis CA, Papadopoulos SP, Campo R, Gordts S, Tarlatzis BC, Grimbizis GF. Clinical implications of congenital uterine anomalies: A meta-analysis of comparative studies. Reprod Biomed Online 2014;29(6):665-83.

29. Yang MJ, Tseng JY, Chen CY, Li HY. Delivery of double singleton pregnancies in a woman with a double uterus, double cervix, and complete septate vagina. J Chinese Med Assoc. 2015;78(12):746-8.

30. Post RJ, Templeman RMB. Twin gestation in a uterus didelphys with only one functional cervix: A case report. Case Reports Women's Heal. 2019;22.

31. Jackson JR, Williams B, Thorp J. Spontaneous Triplets Carried in a Uterus Didelphys. Case Reports Women's Heal. 2014;3-4:1-2.

32. Pankaja S, Ip P OF. Successful Pregnancy with Uterus Didelphys. J Androl Gynecol. 2016;4(2):3.

33. Turocy JM, Rackow BW. Uterine factor in recurrent pregnancy loss. Seminars in Perinatol. 2019;43:74-9.

34. Acien P. Reproductive performance of women with uterine malformations. Hum Reprod. 1193;8(1):1226.

35. Chandrasekhar C. Report of two cases of uterus didelphys and rare ectopic (cornual and cervical) pregnancies. Clin Imaging. 2007;31(1):57-61.

36. Cohen AW, Chhibber G. Obstetric complications of congenital anomalies of the paramesonephric ducts. Semin Reprod Endocrinol. 1986;4(1):59-66.

Cite this article as: Ekanem E, Lalrinawmi L, Sinha A, Abdelrazik T. Pregnancy in a didelphic uterus: a case report of an unusual phenomenon and review of literature. Int J Reprod Contracept Obstet Gynecol 2021;10:1162-8. 